\title{
THRESHOLD APPROXIMATIONS WITH CORRECTOR FOR THE RESOLVENT OF A FACTORIZED SELFADJOINT OPERATOR FAMILY
}

\author{
M. SH. BIRMAN AND T. A. SUSLINA
}

In fond memory of Ol'ga Aleksandrovna Ladyzhenskaya

\begin{abstract}
In a Hilbert space, a family of operators admitting a factorization $A(t)=$ $X(t)^{*} X(t)$, where $X(t)=X_{0}+t X_{1}, t \in \mathbb{R}$, is considered. It is assumed that the subspace $\mathfrak{N}=\operatorname{Ker} A(0)$ is finite-dimensional. For the resolvent $\left(A(t)+\varepsilon^{2} I\right)^{-1}$ with small $\varepsilon$, an approximation in the operator norm is obtained on a fixed interval $|t| \leq t^{0}$. This approximation involves the so-called "corrector"; the remainder term is of order $O(1)$. The results are aimed at applications to homogenization of periodic differential operators in the small period limit. The paper develops and refines the results of Chapter 1 of our paper in St. Petersburg Math. J. 15 (2004), 639-714.
\end{abstract}

\section{INTRODUCTION}

0.1. The present paper is devoted to further development of the operator-theoretic material presented in Chapter 1 of $[\mathrm{BSu}]$. In $[\mathrm{BSu}$, this approach served as a basis for the study of the homogenization effect for periodic differential operators (pDOs) in $L_{2}\left(\mathbb{R}^{d} ; \mathbb{C}^{n}\right)$. Under this approach, the homogenization effect can be studied consistently as a spectral threshold effect near the bottom of the spectrum of a pDO. Namely, after partial diagonalization of a pDO, we arrive at a factorized operator family of the form (1.1) (see below). Preliminarily, such families were studied in [BSu, Chapter 1] with the help of abstract operator theory. More precisely, the resolvent

$$
\left(A(t)+\varepsilon^{2} I\right)^{-1}, \quad \varepsilon>0,
$$

was approximated in terms of the resolvent of the germ $S$ (see Subsection 1.3 below). The germ $S$ is a positive operator acting in the finite-dimensional subspace $\mathfrak{N}=\operatorname{Ker} A(0)$ of dimension $n \geq 1$. For $\varepsilon>0$ and $|t| \leq t^{0}$, the difference of the resolvents was estimated (in the operator norm) by $C \varepsilon^{-1}$. The constants $C$ and $t^{0}$ were well controlled; in general, the order $\varepsilon^{-1}$ in this estimate is sharp. This statement, which is of its own interest, automatically leads to a similar estimate for the norm of the difference of the resolvents of two different families of the form (1.1) having the same germ S. Precisely this latter estimate and its modifications were applied in $[\mathrm{BSu}$ to homogenization problems.

Though these abstract results were aimed at applications to homogenization, formally they are not related to it, pertaining to the spectral perturbation theory of selfadjoint operator families in a Hilbert space.

2000 Mathematics Subject Classification. Primary 47A55.

Key words and phrases. Threshold approximations, homogenization, corrector.

Supported by RFBR (grant no. 05-01-01076). 
0.2. Perturbation theory in the spirit of $[\mathrm{BSu}$, Chapter 1] can be developed further in order to find more accurate finite-dimensional approximations of the resolvent $(*)$. Herewith, we obtain a remainder estimate of order $O(1)$ (instead of $\left.O\left(\varepsilon^{-1}\right)\right)$, which is uniform in $\varepsilon$ and $t$ (under the same conditions on the family $A(t)$ as before). Besides refined finitedimensional approximations of the resolvent, we obtain accurate approximations for it in terms of other operator families. Combined with the corresponding estimates of the remainder terms, such approximations can be applied to homogenization problems for pDOs.

The corresponding abstract considerations now become more involved. Therefore, the authors decided to isolate them in the present paper. Applications to the homogenization of pDOs will be considered elsewhere.

In applications to homogenization, the resulting refined approximation for the resolvent $(*)$ generates correction terms of the order of $\varepsilon$. In homogenization theory, a correction term of the order of $\varepsilon$ is called a corrector. We used this term in the title of the present paper.

Formally speaking, the present paper can be read independently of [BSu, Chapter 1]. All necessary facts from that paper are repeated here, though without proofs. At the same time, the general method of investigation is the same as in [BSu, Chapter 1], so that being acquainted with $[\mathrm{BSu}]$ could facilitate reading.

0.3. The construction of the refined finite-dimensional approximation of the resolvent (*) involves more than the germ $S$. Besides $S$, we need two more operators of finite rank. The first of these is the operator $Z$ defined (together with an auxiliary operator $R$ ) in Subsection 1.2; we have $Z \mathfrak{N} \subset \mathfrak{N}^{\perp}$. The second is the operator $N$ defined in (4.13); we have $N \mathfrak{N} \subset \mathfrak{N}$. In a natural way, the operator $N$ splits: $N=N_{0}+N_{*}$. In the eigenbasis of the operator $S$, the matrix of $N_{0}$ is diagonal. On the contrary, the matrix of $N_{*}$ has zero diagonal (hence, $N_{*}=0$ for $n=1$ ). The operator $N$ is studied in detail in Subsection 4.2 .

The main result (Theorem 5.1) about finite-dimensional approximation of the resolvent $(*)$ is given by (5.21), where $\Xi$ (see (5.5)) is defined in terms of $S$, and the remainder term $\mathcal{J}$ is uniformly bounded in the operator norm. Theorem 5.1 implies Theorem 5.5, where a refined approximation for the resolvent $(*)$ is given in terms of the resolvent of another operator family. The corresponding formula (5.28) also involves the operators $Z$ and $N$.

Theorem 5.5 is convenient for applications to homogenization problems for pDOs. It turns out that the terms in (5.28) that contain the factors $t$ and $t^{3}$ generate the corrector. Note that the corrector traditional for homogenization theory corresponds to the term $t Z\left(\widetilde{A}(t)+\varepsilon^{2} I\right)^{-1}$ in (5.28). At the same time, to obtain the remainder estimate of the order of $\varepsilon^{2}$, we need to take into account all the terms distinguished in (5.28).

Theorems 5.1 and 5.5 are the main results of the paper. However, in the final $\S 6$ we obtain some modifications and generalizations of these theorems, which are also useful for applications to the homogenization of pDOs.

0.4. The paper consists of six sections. In $\S 1$, we list the necessary facts from $\mathrm{BSu}$, Chapter 1]. In $\S \S 2-4$ we obtain preliminary estimates and calculate the operators $Z$ and $N$. As in [BSu, Chapter 1], for estimating the remainder terms we use contour integration, while for calculations we use power series expansions given by spectral analytic perturbation theory. $\S \S 5$ and 6 contain the main results of the paper.

Though the triple enumeration was used in $\mathrm{BSu}$, all our references to that paper concern only Chapter 1. Therefore, we omit the number of the chapter when citing.

Below, the absolute constants are denoted by $\beta$ with various indices. The other constants in estimates are denoted by $c, C, \mathcal{C}$ (also with indices). 


\section{§1. Preliminaries}

For the most part, the content of this section is borrowed from [BSu, Chapter 1].

1.1. Quadratic pencils. We denote by $\mathfrak{H}$ and $\mathfrak{H}_{*}$ separable complex Hilbert spaces; $X_{0}: \mathfrak{H} \rightarrow \mathfrak{H}_{*}$ is a densely defined and closed operator; $X_{1}: \mathfrak{H} \rightarrow \mathfrak{H}_{*}$ is a bounded operator. Then the operator $X(t):=X_{0}+t X_{1}, t \in \mathbb{R}$, is closed on the domain $\operatorname{Dom} X(t)=\operatorname{Dom} X_{0}$. Our main object is the family of selfadjoint positive operators

$$
A(t):=X(t)^{*} X(t), \quad t \in \mathbb{R}
$$

in $\mathfrak{H}$. We denote $A(0)=X_{0}^{*} X_{0}=: A_{0}$. The operator (1.1) corresponds to the quadratic form $a(t)[u, u]=\|X(t) u\|_{\mathfrak{H}_{*}}^{2}, u \in \operatorname{Dom} X_{0}$. Next,

$$
\mathfrak{N}:=\operatorname{Ker} A_{0}=\operatorname{Ker} X_{0}, \quad \mathfrak{N}_{*}:=\operatorname{Ker} X_{0} X_{0}^{*}=\operatorname{Ker} X_{0}^{*} .
$$

It is assumed that the point $\lambda_{0}=0$ is an isolated point of the spectrum of $A_{0}$ (the distance from this point to the rest of the spectrum is denoted by $\left.d^{0}\right)$, and

$$
0<n:=\operatorname{dim} \mathfrak{N}<\infty, \quad n \leq n_{*}:=\operatorname{dim} \mathfrak{N}_{*} \leq \infty .
$$

Let $F(t, s)$ denote the spectral projection of $A(t)$ for the interval $[0, s]$. In what follows, we fix a number $\delta>0$ such that $8 \delta<d^{0}$. It turns out that

$$
F(t, \delta)=F(t, 3 \delta), \quad \operatorname{rank} F(t, \delta)=n, \quad|t| \leq t^{0}=t^{0}(\delta)=\delta^{1 / 2}\left\|X_{1}\right\|^{-1}
$$

(see [BSu, (1.3)]). Let $P$ be the orthogonal projection of $\mathfrak{H}$ onto $\mathfrak{N}$, and let $P_{*}$ be the orthogonal projection of $\mathfrak{H}_{*}$ onto $\mathfrak{N}_{*}$. Next, $P^{\perp}=I-P, \mathfrak{N}^{\perp}=\mathfrak{H} \ominus \mathfrak{N}$, and so on.

1.2. The operators $Z$ and $R$. Let $\mathcal{D}:=\operatorname{Dom} X_{0} \cap \mathfrak{N}^{\perp}$, and let $u \in \mathfrak{H}_{*}$. We consider the following equation for a function $\psi \in \mathcal{D}$ :

$$
\left(X_{0} \psi, X_{0} \zeta\right)_{\mathfrak{H}_{*}}=\left(u, X_{0} \zeta\right)_{\mathfrak{H}_{*}}, \quad \zeta \in \mathcal{D}
$$

(cf. [BSu, (1.7)]). Equation (1.3) admits a unique solution $\psi$, and this solution satisfies $\left\|X_{0} \psi\right\|_{\mathfrak{H}_{*}} \leq\|u\|_{\mathfrak{H}_{*}}$. Now, suppose that

$$
\omega \in \mathfrak{N}, \quad u=-X_{1} \omega
$$

the corresponding solution of (1.3) is denoted by $\psi(\omega)$. We define a bounded operator $Z: \mathfrak{H} \rightarrow \mathfrak{H}$ by the following relations:

$$
Z \omega=\psi(\omega), \omega \in \mathfrak{N} ; \quad Z x=0, \quad x \in \mathfrak{N}^{\perp} .
$$

Clearly, $\operatorname{rank} Z \leq n$. To estimate the norm of $Z$, we put $\zeta=\psi(\omega)$ in the formula

$$
\left(X_{0} \psi(\omega)+X_{1} \omega, X_{0} \zeta\right)_{\mathfrak{H}_{*}}=0, \quad \zeta \in \mathcal{D}
$$

Then

$$
\left(A_{0} \psi(\omega), \psi(\omega)\right)_{\mathfrak{H}}=\left\|X_{0} \psi(\omega)\right\|_{\mathfrak{H}_{*}}^{2} \leq\left\|X_{1}\right\|^{2}\|\omega\|_{\mathfrak{H}}^{2} .
$$

Since $8 \delta\|\zeta\|_{\mathfrak{H}}^{2} \leq\left(A_{0} \zeta, \zeta\right)_{\mathfrak{H}}, \zeta \in \mathcal{D}$, relations (1.5) and (1.7) imply the inequality

$$
\|Z\|_{\mathfrak{H} \rightarrow \mathfrak{H}} \leq(8 \delta)^{-1 / 2}\left\|X_{1}\right\|_{\mathfrak{H} \rightarrow \mathfrak{H}_{*}}=: \mathcal{C}_{1} .
$$

Note that $Z P=Z, P Z=0$.

Now, assuming that (1.4) and (1.6) are satisfied, we put

$$
\omega_{*}=X_{0} \psi(\omega)+X_{1} \omega
$$

We introduce a linear operator $R$ (see [BSu, Subsection 1.2]) as follows:

$$
R: \mathfrak{N} \rightarrow \mathfrak{N}_{*}, \quad R \omega=\omega_{*} \in \mathfrak{N}_{*} .
$$

Another representation for $R$ is given by

$$
R=\left.P_{*} X_{1}\right|_{\mathfrak{N}} .
$$


1.3. The operator $S$ (the germ). The selfadjoint operator

$$
S=R^{*} R: \mathfrak{N} \rightarrow \mathfrak{N}
$$

is called the spectral germ of the operator family (1.1) at $t=0$ (cf. [BSu, Subsection 1.3]). The germ also admits the following representations:

$$
S=\left.P X_{1}^{*} P_{*} X_{1}\right|_{\mathfrak{N}}, \quad(S \zeta, \zeta)_{\mathfrak{H}}=\|R \zeta\|_{\mathfrak{H}_{*}}^{2}=\left\|P_{*} X_{1} \zeta\right\|_{\mathfrak{H}_{*}}^{2}, \quad \zeta \in \mathfrak{N} .
$$

The germ $S$ is said to be nondegenerate if $\operatorname{Ker} S=\{0\}$, or, equivalently, rank $R=n$. Let $\widetilde{A}(t)=\widetilde{X}(t)^{*} \widetilde{X}(t)$ be another operator family in $\mathfrak{H}$ of the form (1.1) subject to the same conditions as $A(t)\left(\widetilde{\mathfrak{H}}_{*}\right.$ may be different from $\left.\mathfrak{H}_{*}\right)$. We say that the families $A(t)$ and $\widetilde{A}(t)$ are threshold equivalent if $\mathfrak{N}=\widetilde{\mathfrak{N}}$ and $S=\widetilde{S}$.

In accordance with the general analytic perturbation theory (see $[\mathrm{K}]$ ), for $|t| \leq t^{0}$ there exist real-analytic functions $\lambda_{l}(t)$ and real-analytic $\mathfrak{H}$-valued functions $\varphi_{l}(t)$ such that

$$
A(t) \varphi_{l}(t)=\lambda_{l}(t) \varphi_{l}(t), \quad l=1, \ldots, n, \quad|t| \leq t^{0}=t^{0}(\delta),
$$

and the functions $\varphi_{l}(t), l=1, \ldots, n$, form an orthonormal basis in $F(t, \delta) \mathfrak{H}$. For sufficiently small $t_{*}$ and $|t| \leq t_{*} \leq t^{0}$, we can write convergent power series expansions:

$$
\begin{aligned}
& \lambda_{l}(t)=\gamma_{l} t^{2}+\mu_{l} t^{3}+\cdots, \quad \gamma_{l} \geq 0, \quad \mu_{l} \in \mathbb{R}, \quad l=1, \ldots, n, \\
& \varphi_{l}(t)=\omega_{l}+t \varphi_{l}^{(1)}+t^{2} \varphi_{l}^{(2)}+\cdots, \quad l=1, \ldots, n .
\end{aligned}
$$

The elements $\omega_{l}=\varphi_{l}(0), l=1, \ldots, n$, form an orthonormal basis in $\mathfrak{N}$. Substituting (1.13) and (1.14) into (1.12) and comparing the coefficients of $t$ and $t^{2}$, we see that

$$
\begin{aligned}
\widetilde{\omega}_{l} & :=\varphi_{l}^{(1)}-\psi\left(\omega_{l}\right)=\varphi_{l}^{(1)}-Z \omega_{l} \in \mathfrak{N}, \quad l=1, \ldots, n, \\
S \omega_{l} & =\gamma_{l} \omega_{l}, \quad l=1, \ldots, n .
\end{aligned}
$$

Relations (1.16) are equivalent to

$$
S P=\sum_{l=1}^{n} \gamma_{l}\left(\cdot, \omega_{l}\right)_{\mathfrak{H}} \omega_{l} .
$$

From (1.17) it follows that the germ $S$ does not depend on a particular factorization of the form (1.1) for $A(t)$. At the same time, representation (1.11) shows that $S$ does not depend on the choice of the basis (1.14) (which may be nonunique). If all the eigenvalues $\gamma_{l}$ in (1.16) are simple, then the elements $\omega_{l}$ in (1.14) are defined in terms of the germ $S$ uniquely (up to phase factors). If there are multiple eigenvalues among $\gamma_{l}$, then the knowledge of $S$ is not sufficient for determining the elements $\omega_{l}$.

In connection with the expansions (1.13) and (1.14), we note the following. Differentiating the relations $\left(\varphi_{k}(t), \varphi_{l}(t)\right)_{\mathfrak{H}}=\delta_{k l}$ and putting $t=0$, we obtain

$$
\left(\varphi_{k}^{(1)}, \omega_{l}\right)_{\mathfrak{H}}+\left(\omega_{k}, \varphi_{l}^{(1)}\right)_{\mathfrak{H}}=0, \quad k, l=1, \ldots, n .
$$

Combining this with $(1.15)$ and the condition $\psi\left(\omega_{k}\right) \in \mathfrak{N}^{\perp}$, we see that

$$
\left(\widetilde{\omega}_{k}, \omega_{l}\right)_{\mathfrak{H}}+\left(\omega_{k}, \widetilde{\omega}_{l}\right)_{\mathfrak{H}}=0, \quad k, l=1, \ldots, n .
$$

\section{§. Approximation of the projection $F(t, \delta)$}

2.1. Notation. The contour $\Gamma$. Sometimes, we use the brief notation $F(t)=F(t, \delta)$. We denote by $R_{z}(t)$ the resolvent $(A(t)-z I)^{-1}$ of the operator $A(t)$ and by $R_{z}(0)$ the resolvent of $A_{0}$. The operator-valued function $F(t)$ is real-analytic for $|t| \leq t^{0}$. If $|t| \leq t_{*}$, this function admits a power series expansion

$$
F(t)=P+t F_{1}+\cdots
$$


convergent in the operator norm. Now, for us it suffices to estimate the difference

$$
F_{2}(t):=F(t)-P-t F_{1}, \quad|t| \leq t^{0}(\delta)=\delta^{1 / 2}\left\|X_{1}\right\|^{-1},
$$

and to give an explicit expression for the operator $F_{1}$. It is convenient to obtain the required estimate by integrating the difference of the resolvents over an appropriate contour, while for calculating $F_{1}$ it is more convenient to use the expansions (1.14).

Let $\Gamma \subset \mathbb{C}$ be the contour that envelops the interval $[0, \delta]$ equidistantly at the distance $\delta$. By (1.2), the distance from $\Gamma$ to the spectrum of $A(t)$ is at least $\delta$, whence

$$
\left\|R_{z}(t)\right\| \leq \delta^{-1}, \quad z \in \Gamma, \quad|t| \leq t^{0}(\delta) .
$$

We shall use the following elementary identity:

$$
F(t)-P=\frac{1}{2 \pi i} \int_{\Gamma}\left(R_{z}(0)-R_{z}(t)\right) d z, \quad|t| \leq t^{0}(\delta) .
$$

Note that the usual resolvent identity for the difference of resolvents is not applicable under our assumptions, because, in general, Dom $A(t)$ depends on $t$. However, for the forms $a(t)$ we have $\operatorname{Dom} a(t)=\operatorname{Dom} X(t)=\operatorname{Dom} X_{0}$. This allows us to use a certain analog of the resolvent identity (cf. [BSu, (2.13)] and Subsection 3.1 below). However, so far it suffices to use a "finished" result of [BSu].

2.2. The estimate for the norm of $F_{2}(t)$. The following result was obtained in BSu, $\S 3$, Theorem 3.1]. Now we reproduce this result in detail.

Theorem 2.1. Let $z \in \Gamma$, and let $|t| \leq t^{0}(\delta)$. Then

$$
R_{z}(0)-R_{z}(t)=t \mathcal{I}_{1}^{0}+t^{2} \mathcal{I}_{2}^{+}+\Psi_{0}(t),
$$

where $\Psi_{0}(t)$ satisfies the estimate

$$
\left\|\Psi_{0}(t)\right\| \leq \beta^{(0)}|t|^{3} \delta^{-5 / 2}\left\|X_{1}\right\|^{3},
$$

and the bounded operators $\mathcal{I}_{1}^{0}$ and $\mathcal{I}_{2}^{+}$do not depend on $t$. For the terms $\mathcal{I}_{1}^{0}$ and $\mathcal{I}_{2}^{+}$we have

$$
\begin{aligned}
\mathcal{I}_{1}^{0} & =\Omega_{z}(0) T_{\delta}^{(1)} R_{z}(0) \\
\mathcal{I}_{2}^{+} & =\Omega_{z}(0) T_{\delta}^{(2)} R_{z}(0)-\Omega_{z}(0) T_{\delta}^{(1)} \Omega_{z}(0) T_{\delta}^{(2)} R_{z}(0)
\end{aligned}
$$

Here

$$
\Omega_{z}(t):=I+(z+2 \delta) R_{z}(t),
$$

and we have

$$
\left\|\Omega_{z}(t)\right\| \leq 5, \quad z \in \Gamma, \quad|t| \leq t^{0}(\delta) .
$$

The definition of the operators $T_{\delta}^{(1)}$ and $T_{\delta}^{(2)}$ is more complicated. On Dom $X_{0}$ we introduce the metric form $\left\|X_{0} u\right\|_{\mathfrak{H}_{*}}^{2}+2 \delta\|u\|_{\mathfrak{H}^{2}}^{2}, u \in \operatorname{Dom} X_{0}$. The Hilbert space Dom $X_{0}$ with this metric form is denoted by $\mathfrak{d}$. The operators $T_{\delta}^{(1)}$ and $T_{\delta}^{(2)}$ are selfadjoint continuous operators in $\mathfrak{d}$ generated by the forms $2 \operatorname{Re}\left(X_{0} u, X_{1} u\right)_{\mathfrak{H}_{*}}$ and $\left\|X_{1} u\right\|_{\mathfrak{H}_{*}}^{2}, u \in \mathfrak{d}$, respectively. The norms of $T_{\delta}^{(1)}$ and $T_{\delta}^{(2)}$ in $\mathfrak{d}$ satisfy

$$
\left\|T_{\delta}^{(1)}\right\|_{\mathfrak{o} \rightarrow \mathfrak{d}} \leq(2 \delta)^{-1 / 2}\left\|X_{1}\right\|, \quad\left\|T_{\delta}^{(2)}\right\|_{\mathfrak{o} \rightarrow \mathfrak{d}} \leq(2 \delta)^{-1}\left\|X_{1}\right\|^{2} .
$$

For estimating $F_{2}(t)$, we need a rough version of Theorem 2.1: for $\mathcal{I}_{2}^{+}$it suffices to have only an estimate. This estimate follows from the representation (2.6) by an automatic application of the results of [BSu, Subsections 2.2 and 3.2]. We shall not enter into the details here. It is easy to reconstruct the details on the basis of $\S 3$, where 
more complicated cases will be discussed. It is easily seen that $\left\|\mathcal{I}_{2}^{+}\right\| \leq 75 \delta^{-2}\left\|X_{1}\right\|^{2}$. Combining this with (2.4), we obtain

$$
\begin{aligned}
\left\|t^{2} \mathcal{I}_{2}^{+}+\Psi_{0}(t)\right\| & \leq 75 \delta^{-2}\left\|X_{1}\right\|^{2} t^{2}+\beta^{(0)} \delta^{-5 / 2}\left\|X_{1}\right\|^{3}|t|^{3} \\
& \leq \beta^{(1)} \delta^{-2}\left\|X_{1}\right\|^{2} t^{2}, \quad|t| \leq t^{0}(\delta) .
\end{aligned}
$$

Thus, instead of (2.3) and (2.4) we obtain the following representation:

$$
R_{z}(0)-R_{z}(t)=t \mathcal{I}_{1}^{0}+\Psi_{1}(t), \quad\left\|\Psi_{1}(t)\right\| \leq \beta^{(1)} \delta^{-2}\left\|X_{1}\right\|^{2} t^{2},
$$

for $|t| \leq t^{0}(\delta)$. Now (2.2) directly implies the relations

$$
\begin{gathered}
F(t)=P+t F_{1}+F_{2}(t), \quad\left\|F_{2}(t)\right\| \leq \beta^{(2)} \delta^{-1}\left\|X_{1}\right\|^{2} t^{2}, \quad|t| \leq t^{0}(\delta), \\
2 \pi i F_{1}=\int_{\Gamma} \mathcal{I}_{1}^{0} d z .
\end{gathered}
$$

2.3. Calculation of the operator $F_{1}$. This can be done on the basis of (2.5) and (2.11), but we prefer to use the power series expansions (2.1) and (1.14). We put

$$
\psi_{l}=\psi\left(\omega_{l}\right)=Z \omega_{l}, \quad l=1, \ldots, n .
$$

Then, by (1.14) and (1.15),

$$
\begin{aligned}
F(t) & =\sum_{l=1}^{n}\left(\cdot, \varphi_{l}(t)\right)_{\mathfrak{H}} \varphi_{l}(t) \\
& =\sum_{l=1}^{n}\left(\cdot, \omega_{l}\right)_{\mathfrak{H}} \omega_{l}+t \sum_{l=1}^{n}\left(\left(\cdot, \varphi_{l}^{(1)}\right)_{\mathfrak{H}} \omega_{l}+\left(\cdot, \omega_{l}\right)_{\mathfrak{H}} \varphi_{l}^{(1)}\right)+O\left(t^{2}\right) \\
& =P+t \sum_{l=1}^{n}\left(\left(\cdot, \psi_{l}\right)_{\mathfrak{H}} \omega_{l}+\left(\cdot, \omega_{l}\right)_{\mathfrak{H}} \psi_{l}\right)+t Y+O\left(t^{2}\right),
\end{aligned}
$$

where $Y:=\sum_{l=1}^{n}\left(\left(\cdot, \widetilde{\omega}_{l}\right)_{\mathfrak{H}} \omega_{l}+\left(\cdot, \omega_{l}\right)_{\mathfrak{H}} \widetilde{\omega}_{l}\right)$. Clearly, $\left.Y\right|_{\mathfrak{N}^{\perp}}=0$. Relation (1.18) implies that $\left(Y \omega_{j}, \omega_{k}\right)=0, j, k=1, \ldots, n$, whence $\left.Y\right|_{\mathfrak{N}}=0$. Thus, $Y=0$, and from (2.1) and (2.13) we deduce that

$$
F_{1}=\sum_{l=1}^{n}\left(\left(\cdot, \psi_{l}\right)_{\mathfrak{H}} \omega_{l}+\left(\cdot, \omega_{l}\right)_{\mathfrak{H}} \psi_{l}\right)
$$

Formula (2.14) can be rewritten in an invariant form. Indeed,

$$
\sum_{l=1}^{n}\left(u, \omega_{l}\right)_{\mathfrak{H}} \psi_{l}=Z \sum_{l=1}^{n}\left(u, \omega_{l}\right)_{\mathfrak{H}} \omega_{l}=Z P u, \quad u \in \mathfrak{H} .
$$

Thus, (2.14) is equivalent to the relation

$$
F_{1}=Z P+P Z^{*} .
$$

We arrive at the following theorem.

Theorem 2.2. In the representation (2.10), the operator $F_{1}$ is defined by (2.15).

Note that $Z=F_{1} P$. It follows that $Z$ (together with $F_{1}$ and $P$ ) is determined directly by the family $A(t)$ and does not depend on the factorization (1.1). 


\section{§3. REFINED APPROXIMATION FOR THE DIFFERENCE OF THE RESOLVENTS ON THE CONTOUR $\Gamma$}

3.1. The resolvent identity. We write the resolvent identity, using the notation of Subsection 2.2. Initially, this identity looks like the following (see [BSu, (3.11)]):

$$
\mathcal{I}:=R_{z}(0)-R_{z}(t)=\Omega_{z}(0) T_{\delta}(t) R_{z}(t)
$$

where

$$
T_{\delta}(t)=t T_{\delta}^{(1)}+t^{2} T_{\delta}^{(2)} .
$$

We need to iterate identity (3.1) in order to separate three terms from $\mathcal{I}$ (but not two terms, as in (2.3)). We are not interested in the separated terms themselves. Our goal is to estimate the remainder term. Therefore, below we shall use the symbol " " (together with "="), omitting the terms of the first three orders with coefficients independent of $t$. Iterating (3.1), we obtain

$$
\begin{gathered}
\mathcal{I}=\Omega_{z}(0) T_{\delta}(t) R_{z}(0)-\Omega_{z}(0) T_{\delta}(t) \mathcal{I} \sim-\Omega_{z}(0) T_{\delta}(t) \Omega_{z}(0) T_{\delta}(t) R_{z}(t) \\
=-t^{2} \Omega_{z}(0) T_{\delta}^{(1)} \Omega_{z}(0) T_{\delta}^{(1)} R_{z}(t) \\
-t^{3} \Omega_{z}(0)\left(T_{\delta}^{(2)} \Omega_{z}(0) T_{\delta}^{(1)}+T_{\delta}^{(1)} \Omega_{z}(0) T_{\delta}^{(2)}\right) R_{z}(t)+t^{4} \Theta_{1}(t) \\
\Theta_{1}(t)=-\Omega_{z}(0) T_{\delta}^{(2)} \Omega_{z}(0) T_{\delta}^{(2)} R_{z}(t) .
\end{gathered}
$$

Now we perform yet another iteration in the terms with $t^{2}$ and $t^{3}$ on the right-hand side of (3.3), obtaining

$$
\begin{aligned}
\mathcal{I} & \sim t^{2} \Omega_{z}(0) T_{\delta}^{(1)} \Omega_{z}(0) T_{\delta}^{(1)} \Omega_{z}(0) T_{\delta}(t) R_{z}(t)+t^{3} \Theta_{3}(t)+t^{4} \Theta_{1}(t) \\
& =t^{3} \Omega_{z}(0) T_{\delta}^{(1)} \Omega_{z}(0) T_{\delta}^{(1)} \Omega_{z}(0) T_{\delta}^{(1)} R_{z}(t)+t^{4} \Theta_{2}(t)+t^{3} \Theta_{3}(t)+t^{4} \Theta_{1}(t), \\
& \Theta_{2}(t)=\Omega_{z}(0) T_{\delta}^{(1)} \Omega_{z}(0) T_{\delta}^{(1)} \Omega_{z}(0) T_{\delta}^{(2)} R_{z}(t), \\
& \Theta_{3}(t)=\Omega_{z}(0)\left(T_{\delta}^{(2)} \Omega_{z}(0) T_{\delta}^{(1)}+T_{\delta}^{(1)} \Omega_{z}(0) T_{\delta}^{(2)}\right) \Omega_{z}(0) T_{\delta}(t) R_{z}(t) .
\end{aligned}
$$

Finally, we iterate the first term on the right in (3.5) once again. This yields

$$
\begin{aligned}
\mathcal{I} & \sim t^{3}\left(\Theta_{3}(t)+\Theta_{4}(t)\right)+t^{4}\left(\Theta_{1}(t)+\Theta_{2}(t)\right) \\
\Theta_{4}(t) & =-\Omega_{z}(0) T_{\delta}^{(1)} \Omega_{z}(0) T_{\delta}^{(1)} \Omega_{z}(0) T_{\delta}^{(1)} \Omega_{z}(0) T_{\delta}(t) R_{z}(t) .
\end{aligned}
$$

3.2. Estimates for $\Theta_{j}(t), j=1,2,3,4$, on the contour $\Gamma$. Below we always assume that $z \in \Gamma$ and $|t| \leq t^{0}(\delta)=\delta^{1 / 2}\left\|X_{1}\right\|^{-1}$. We lean upon estimates (2.8) and (2.9). Note also that (2.9) and the inequality $|t| \leq t^{0}(\delta)$ imply the following estimate for the operator $(3.2)$ :

$$
\left\|T_{\delta}(t)\right\|_{\mathfrak{d} \rightarrow \mathfrak{d}} \leq 2^{-1}(\sqrt{2}+1)|t| \delta^{-1 / 2}\left\|X_{1}\right\| .
$$

Also, we need the following auxiliary inequalities. Let $L, L_{1}$, and $L_{2}$ be continuous operators in $\mathfrak{d}$. Then

$$
\begin{aligned}
&\left\|L R_{z}(0)\right\|_{\mathfrak{H} \rightarrow \mathfrak{H}} \leq \beta^{(3)} \delta^{-1}\|L\|_{\mathfrak{d} \rightarrow \mathfrak{d}}, \\
&\left\|L_{1} \Omega_{z}(0) L_{2} R_{z}(t)\right\|_{\mathfrak{H} \rightarrow \mathfrak{H}} \leq \beta^{(4)} \delta^{-1}\left\|L_{1}\right\|_{\mathfrak{d} \rightarrow \mathfrak{d}}\left\|L_{2}\right\|_{\mathfrak{d} \rightarrow \mathfrak{d}},
\end{aligned}
$$

which follows directly from $[\mathrm{BSu},(2.16),(2.17)]$ if we put $A=A(0), B=A(t), \gamma=2 \delta$, $\alpha=\sqrt{2}$, and use (2.8) and the inequality $|z+2 \delta| \leq 4 \delta$ on $\Gamma$. 
Theorem 3.1. If $z \in \Gamma$ and $|t| \leq t^{0}(\delta)$, then the norms of the operators (3.4), (3.6), (3.7), and (3.9) in $\mathfrak{H}$ satisfy the estimates

$$
\begin{array}{ll}
\left\|\Theta_{1}(t)\right\| \leq \beta_{1} \delta^{-3}\left\|X_{1}\right\|^{4}, \quad\left\|\Theta_{2}(t)\right\| \leq \beta_{2} \delta^{-3}\left\|X_{1}\right\|^{4}, \\
\left\|\Theta_{3}(t)\right\| \leq \beta_{3}|t| \delta^{-3}\left\|X_{1}\right\|^{4}, \quad\left\|\Theta_{4}(t)\right\| \leq \beta_{4}|t| \delta^{-3}\left\|X_{1}\right\|^{4} .
\end{array}
$$

Proof. All four inequalities are proved in a similar way. We prove the last of them, which requires more effort. By (2.8), the left factor in (3.9) can be ignored. By (2.7), the question reduces to estimating the operator

$$
\begin{gathered}
\mathcal{Y}_{1}(t)+(z+2 \delta) \mathcal{Y}_{2} \mathcal{Y}_{3}(t) \\
\mathcal{Y}_{1}(t)=T_{\delta}^{(1)} \Omega_{z}(0)\left(T_{\delta}^{(1)}\right)^{2} \Omega_{z}(0) T_{\delta}(t) R_{z}(t), \\
\mathcal{Y}_{2}=T_{\delta}^{(1)} \Omega_{z}(0) T_{\delta}^{(1)} R_{z}(0), \quad \mathcal{Y}_{3}(t)=T_{\delta}^{(1)} \Omega_{z}(0) T_{\delta}(t) R_{z}(t) .
\end{gathered}
$$

We estimate $\mathcal{Y}_{1}(t)$. Using (2.7) once again, we get

$$
\mathcal{Y}_{1}(t)=\left(T_{\delta}^{(1)}\right)^{3} \Omega_{z}(0) T_{\delta}(t) R_{z}(t)+(z+2 \delta) T_{\delta}^{(1)} R_{z}(0)\left(T_{\delta}^{(1)}\right)^{2} \Omega_{z}(0) T_{\delta}(t) R_{z}(t) .
$$

The first summand in (3.16) is estimated with the help of (3.12), and the second with the help of (3.11) and (3.12). Also, we use estimates (2.9) and (3.10). Both summands give similar contributions, and for the operator (3.14) we obtain

$$
\left\|\mathcal{Y}_{1}(t)\right\| \leq \beta^{(5)}|t| \delta^{-3}\left\|X_{1}\right\|^{4} .
$$

Each of the two operators (3.15) is of the form (3.12), which implies that the second summand in (3.13) is also dominated by $\beta^{(6)}|t| \delta^{-3}\left\|X_{1}\right\|^{4}$. The required estimate for $\left\|\Theta_{4}(t)\right\|$ is proved.

3.3. Representation for the difference of the resolvents. Such a representation follows directly from (3.8) and Theorem 3.1.

Theorem 3.2. If $z \in \Gamma$ and $|t| \leq t^{0}(\delta)$, then

$$
\begin{gathered}
R_{z}(0)-R_{z}(t)=t \mathcal{I}_{1}^{0}+t^{2} \mathcal{I}_{2}^{+}+t^{3} \mathcal{I}_{3}+\Psi_{*}(t), \\
\left\|\Psi_{*}(t)\right\| \leq \beta_{*} t^{4} \delta^{-3}\left\|X_{1}\right\|^{4},
\end{gathered}
$$

and the operators $\mathcal{I}_{1}^{0}, \mathcal{I}_{2}^{+}$, and $\mathcal{I}_{3}$ are continuous in $\mathfrak{H}$ and do not depend on $t$.

\section{§4. Approximation for the operator-valued function $A(t) F(t)$}

4.1. Application of Theorem 3.2. Using Theorem 3.2 and the elementary formula

$$
A(t) F(t)=\frac{1}{2 \pi i} \int_{\Gamma} z\left(R_{z}(0)-R_{z}(t)\right) d z
$$

we obtain the following statement.

Theorem 4.1. For $|t| \leq t^{0}(\delta)$, we have

$$
A(t) F(t)=t^{2} S P+t^{3} K+\Psi(t)
$$

and

$$
\|\Psi(t)\| \leq \beta t^{4} \delta^{-1}\left\|X_{1}\right\|^{4},
$$

where the operator $K$ is continuous in $\mathfrak{H}$ and does not depend on $t$.

Note that, in accordance with $\mathrm{BSu}$, when calculating the integral (4.1), for the operators $\mathcal{I}_{1}^{0}$ and $\mathcal{I}_{2}^{+}$in $(3.17)$ we obtain $\mathbb{O}$ and $S P$, respectively. We must calculate the operator $K$. For this, below we use power series expansions. 
4.2. Calculation of the operator $K$. By (1.12)-(1.15) and (2.12), for sufficiently small $t_{*}>0$ and $|t| \leq t_{*}$, we have

$$
\begin{aligned}
A(t) F(t) & =\sum_{l=1}^{n} \lambda_{l}(t)\left(\cdot, \varphi_{l}(t)\right)_{\mathfrak{H}} \varphi_{l}(t) \\
& =\sum_{l=1}^{n}\left(\gamma_{l} t^{2}+\mu_{l} t^{3}\right)\left(\cdot, \omega_{l}+t\left(\psi_{l}+\widetilde{\omega}_{l}\right)\right)_{\mathfrak{H}}\left(\omega_{l}+t\left(\psi_{l}+\widetilde{\omega}_{l}\right)\right)+O\left(t^{4}\right) \\
& =t^{2} S P+t^{3} K+O\left(t^{4}\right) .
\end{aligned}
$$

This implies that the operator $K$ in (4.2) admits the following representation:

$$
\begin{aligned}
K & =K_{0}+N_{0}+N_{*}=K_{0}+N, \\
K_{0} & =\sum_{l=1}^{n} \gamma_{l}\left(\left(\cdot, \psi_{l}\right)_{\mathfrak{H}} \omega_{l}+\left(\cdot, \omega_{l}\right)_{\mathfrak{H}} \psi_{l}\right), \\
N_{0} & =\sum_{l=1}^{n} \mu_{l}\left(\cdot, \omega_{l}\right)_{\mathfrak{H}} \omega_{l}, \\
N_{*} & =\sum_{l=1}^{n} \gamma_{l}\left(\left(\cdot, \widetilde{\omega}_{l}\right)_{\mathfrak{H}} \omega_{l}+\left(\cdot, \omega_{l}\right)_{\mathfrak{H}} \widetilde{\omega}_{l}\right) .
\end{aligned}
$$

Our next goal is to find an invariant representation for $K$. This is easy to do for $K_{0}$ : by (2.12) and (1.17), we have

$$
\sum_{l=1}^{n} \gamma_{l}\left(\cdot, \omega_{l}\right)_{\mathfrak{H}} \psi_{l}=Z \sum_{l=1}^{n} \gamma_{l}\left(\cdot, \omega_{l}\right)_{\mathfrak{H}} \omega_{l}=Z S P,
$$

whence

$$
K_{0}=Z S P+S P Z^{*}
$$

Now we calculate the matrix entries of the operator $N$ in the basis $\left\{\omega_{l}\right\}_{1}^{n}$. Direct inspection yields

$$
\left(N_{*} \omega_{j}, \omega_{k}\right)_{\mathfrak{H}}=\gamma_{k}\left(\omega_{j}, \widetilde{\omega}_{k}\right)_{\mathfrak{H}}+\gamma_{j}\left(\widetilde{\omega}_{j}, \omega_{k}\right)_{\mathfrak{H}}, \quad j, k=1, \ldots, n .
$$

In particular, by (1.18),

$$
\left(N_{*} \omega_{k}, \omega_{k}\right)_{\mathfrak{H}}=0, \quad k=1, \ldots, n
$$

Next,

$$
\left(N_{0} \omega_{j}, \omega_{k}\right)_{\mathfrak{H}}=\delta_{j k} \mu_{k}, \quad j, k=1, \ldots, n .
$$

Finally, combining (4.6) and (4.7) with (1.18), we obtain

$$
\begin{aligned}
\left(N \omega_{j}, \omega_{k}\right)_{\mathfrak{H}} & =\delta_{j k} \mu_{k}+\gamma_{k}\left(\omega_{j}, \widetilde{\omega}_{k}\right)_{\mathfrak{H}}+\gamma_{j}\left(\widetilde{\omega}_{j}, \omega_{k}\right)_{\mathfrak{H}} \\
& =\mu_{k} \delta_{j k}-\gamma_{j}\left(\omega_{j}, \widetilde{\omega}_{k}\right)_{\mathfrak{H}}-\gamma_{k}\left(\widetilde{\omega}_{j}, \omega_{k}\right)_{\mathfrak{H}}, \quad j, k=1, \ldots, n .
\end{aligned}
$$

4.3. Calculation of the operator $N$. The calculation of $N$ is based on the relation

$$
\left(A(t) \varphi_{j}(t), \varphi_{k}(t)\right)_{\mathfrak{H}}=\delta_{j k} \lambda_{j}(t), \quad j, k=1, \ldots, n .
$$

We write the left-hand side of (4.9) as

$$
\left(\left(X_{0}+t X_{1}\right) \varphi_{j}(t),\left(X_{0}+t X_{1}\right) \varphi_{k}(t)\right)_{\mathfrak{H}_{*}} .
$$

Using expansion (1.14) and recalling (1.9), (1.10), and (1.15), we obtain

$$
\left(X_{0}+t X_{1}\right) \varphi_{l}(t)=t\left(R \omega_{l}+t X_{1} \varphi_{l}^{(1)}+t X_{0} \varphi_{l}^{(2)}+\cdots\right) .
$$


We substitute this in (4.10) (with $l=j, k$ ) and look at the total coefficient of $t^{3}$. Since $X_{0}^{*} R=0$, the terms with $\varphi_{l}^{(2)}$ disappear; next, we have $\varphi_{l}^{(1)}=\psi_{l}+\widetilde{\omega}_{l}$. Thus, the coefficient of $t^{3}$ on the left-hand side of (4.9) is equal to

$$
\Delta_{j k}:=\left(R \omega_{j}, X_{1}\left(\psi_{k}+\widetilde{\omega}_{k}\right)\right)_{\mathfrak{H}_{*}}+\left(X_{1}\left(\psi_{j}+\widetilde{\omega}_{j}\right), R \omega_{k}\right)_{\mathfrak{H}_{*}} .
$$

Using (2.12) and the identity $X_{0}^{*} R=0$, we rewrite this coefficient as

$$
\begin{aligned}
\Delta_{j k}= & \left(R \omega_{j}, X_{1} Z \omega_{k}\right)_{\mathfrak{H}_{*}}+\left(X_{1} Z \omega_{j}, R \omega_{k}\right)_{\mathfrak{H}_{*}} \\
& +\left(R \omega_{j}, X_{0} \psi\left(\widetilde{\omega}_{k}\right)+X_{1} \widetilde{\omega}_{k}\right)_{\mathfrak{H}_{*}}+\left(X_{0} \psi\left(\widetilde{\omega}_{j}\right)+X_{1} \widetilde{\omega}_{j}, R \omega_{k}\right)_{\mathfrak{H}_{*}} \\
= & \left(R \omega_{j}, X_{1} Z \omega_{k}\right)_{\mathfrak{H}_{*}}+\left(X_{1} Z \omega_{j}, R \omega_{k}\right)_{\mathfrak{H}_{*}}+\left(R \omega_{j}, R \widetilde{\omega}_{k}\right)_{\mathfrak{H}_{*}}+\left(R \widetilde{\omega}_{j}, R \omega_{k}\right)_{\mathfrak{H}_{*}} .
\end{aligned}
$$

Since $R^{*} R=S$ and $S \omega_{l}=\gamma_{l} \omega_{l}$, the last two summands on the right in (4.11) reduce to $\gamma_{j}\left(\omega_{j}, \widetilde{\omega}_{k}\right)_{\mathfrak{H}}+\gamma_{k}\left(\widetilde{\omega}_{j}, \omega_{k}\right)_{\mathfrak{H}}$, or, by (4.8), to $\mu_{k} \delta_{j k}-\left(N \omega_{j}, \omega_{k}\right)_{\mathfrak{H}}$. Thus, the coefficient of $t^{3}$ on the left-hand side of (4.9) is

$$
\Delta_{j k}=\left(Z^{*} X_{1}^{*} R P \omega_{j}, \omega_{k}\right)_{\mathfrak{H}}+\left((R P)^{*} X_{1} Z \omega_{j}, \omega_{k}\right)_{\mathfrak{H}}+\mu_{k} \delta_{j k}-\left(N \omega_{j}, \omega_{k}\right)_{\mathfrak{H}},
$$

while the coefficient on the right-hand side is obviously equal to $\mu_{k} \delta_{j k}$. So,

$$
\left(N \omega_{j}, \omega_{k}\right)_{\mathfrak{H}}=\left(Z^{*} X_{1}^{*} R P \omega_{j}, \omega_{k}\right)_{\mathfrak{H}}+\left((R P)^{*} X_{1} Z \omega_{j}, \omega_{k}\right)_{\mathfrak{H}}, \quad j, k=1, \ldots, n,
$$

which is equivalent to

$$
N=Z^{*} X_{1}^{*} R P+(R P)^{*} X_{1} Z .
$$

Indeed, the operators on both sides of (4.13) take $\mathfrak{N}$ to $\mathfrak{N}$ and are equal to zero on $\mathfrak{N}^{\perp}$. Therefore, (4.12) implies (4.13).

4.4. The final result. Combining Theorem 4.1 with relations (4.4), (4.5), and (4.13), we arrive at the following theorem.

Theorem 4.2. Under the conditions of Theorem 4.1, the operator $K$ is given by (4.4), where $K_{0}$ and $N$ are defined by (4.5) and (4.13).

Remark 4.3. a) The operator $N$ takes $\mathfrak{N}$ to $\mathfrak{N}$. On the contrary, we have $K_{0} \mathfrak{N} \subset \mathfrak{N}^{\perp}$. Indeed, by (4.5),

$$
P K_{0} P=0 .
$$

b) In the basis $\left\{\omega_{l}\right\}_{1}^{n}$ the operator $N_{0}$ is diagonal, and the matrix of $N_{*}$ has zero diagonal. In particular, $N_{*}=0$ for $n=1$.

c) If $Z=0$, then, by (2.15), (4.5), and (4.13), we have $F_{1}=0$ in (2.10) and $K=$ $K_{0}+N=0$ in $(4.2)$.

In conclusion, we supplement (1.8) with the following elementary estimates:

$$
\begin{gathered}
\|R\| \leq\left\|X_{1}\right\|, \quad\|S\|=\|R\|^{2} \leq\left\|X_{1}\right\|^{2}, \\
\left\|F_{1}\right\| \leq(2 \delta)^{-1 / 2}\left\|X_{1}\right\|, \quad\left\|K_{0}\right\| \leq(2 \delta)^{-1 / 2}\left\|X_{1}\right\|^{3}, \\
\|N\| \leq(2 \delta)^{-1 / 2}\left\|X_{1}\right\|^{3}, \quad\|K\| \leq 2(2 \delta)^{-1 / 2}\left\|X_{1}\right\|^{3} .
\end{gathered}
$$

§5. Approximation of the Resolvent $\left(A(t)+\varepsilon^{2} I\right)^{-1}$ For Small $\varepsilon$

Here we obtain approximations for the resolvent $R_{-\varepsilon^{2}}(t)$ as $\varepsilon \rightarrow 0$ in terms of the germ $S$, the operator $Z$, and other asymptotic characteristics of the family $A(t)$ at $t=0$. The approximating operators have finite rank and depend on two parameters $t$ and $\varepsilon$. The corresponding error estimates are uniform for $|t| \leq t^{0}(\delta)$ and $\varepsilon>0$. 
5.1. The resolvent identity for $A(t)$ and $t^{2} S P$. The following identity can be checked easily:

$$
\begin{aligned}
\mathcal{G}:= & F(t)\left(A(t)+\varepsilon^{2} I\right)^{-1} P=F(t)\left(t^{2} S P+\varepsilon^{2} I\right)^{-1} P \\
& -F(t)\left(A(t)+\varepsilon^{2} I\right)^{-1}\left(A(t) F(t)-t^{2} S P\right)\left(t^{2} S P+\varepsilon^{2} I\right)^{-1} P .
\end{aligned}
$$

(This relation was used in [BSu, Subsection 5.2].) In what follows, it is always assumed that $|t| \leq t^{0}(\delta)=\delta^{1 / 2}\left\|X_{1}\right\|^{-1}, \varepsilon>0$. Moreover, we assume that

$$
A(t) \geq c_{*} t^{2} I, \quad|t| \leq t^{0}(\delta),
$$

for some constant $c_{*}>0$ (cf. [BSu, (5.1)]). Consequently (see [BSu, (5.4), (5.5)]),

$$
\begin{aligned}
\left\|\left(A(t)+\varepsilon^{2} I\right)^{-1} F(t)\right\| & \leq\left(c_{*} t^{2}+\varepsilon^{2}\right)^{-1}, \quad|t| \leq t^{0}(\delta), \\
\left\|\left(t^{2} S P+\varepsilon^{2} I\right)^{-1} P\right\| & \leq\left(c_{*} t^{2}+\varepsilon^{2}\right)^{-1} .
\end{aligned}
$$

By (4.2), we have $A(t) F(t)-t^{2} S P=t^{3} K+\Psi(t)$, where the operator $K$ is defined by (4.4), (4.5), and (4.13), and the operator-valued function $\Psi(t)$ satisfies (4.3). Temporarily, we introduce the notation

$$
\Xi:=\left(t^{2} S P+\varepsilon^{2} I\right)^{-1} P, \quad \mathcal{J}_{1}:=-F(t)\left(A(t)+\varepsilon^{2} I\right)^{-1} \Psi(t) \Xi .
$$

Then (5.1) takes the form

$$
\mathcal{G}=F(t) \Xi-t^{3} F(t)\left(A(t)+\varepsilon^{2} I\right)^{-1} K \Xi+\mathcal{J}_{1} .
$$

The following estimate is borrowed from [BSu, (5.9)]:

$$
\begin{gathered}
\left\|F(t)\left(A(t)+\varepsilon^{2} I\right)^{-1}-\Xi\right\| \leq \mathcal{C}_{0}|t|\left(c_{*} t^{2}+\varepsilon^{2}\right)^{-1}, \\
\mathcal{C}_{0}=\beta_{5}\left(t^{0}(\delta)\right)^{-1}+\beta_{6} c_{*}^{-1} \delta\left(t^{0}(\delta)\right)^{-3} .
\end{gathered}
$$

Then we can rewrite (5.6) as

$$
\mathcal{G}=F(t) \Xi-t^{3} \Xi K \Xi+\mathcal{J}_{1}+\mathcal{J}_{2},
$$

where

$$
\mathcal{J}_{2}=-t^{3}\left(F(t)\left(A(t)+\varepsilon^{2} I\right)^{-1}-\Xi\right) K \Xi .
$$

By (4.4) and (4.14), we can replace $K$ with $N$ in (5.9), where the operator $N$ is defined by (4.13). Thus,

$$
\mathcal{G}=F(t) \Xi-t^{3} \Xi N \Xi+\mathcal{J}_{1}+\mathcal{J}_{2} .
$$

5.2. Further transformations and estimates. First, we estimate the operators $\mathcal{J}_{1}$ and $\mathcal{J}_{2}$ defined by (5.5) and (5.10). From (4.3), (5.3), and (5.4) we deduce that

$$
\left\|\mathcal{J}_{1}\right\| \leq \beta t^{4}\left(c_{*} t^{2}+\varepsilon^{2}\right)^{-2} \delta^{-1}\left\|X_{1}\right\|^{4} \leq \beta c_{*}^{-2} \delta^{-1}\left\|X_{1}\right\|^{4}=: C_{1} .
$$

Next, in a similar way, (4.16), (5.4), and (5.7) imply the inequality

$$
\left\|\mathcal{J}_{2}\right\| \leq \sqrt{2} \mathcal{C}_{0} c_{*}^{-2} \delta^{-1 / 2}\left\|X_{1}\right\|^{3}=: C_{2} ;
$$

here $\mathcal{C}_{0}$ is defined by (5.8).

Now we approximate $F(t)$ on the right-hand side of (5.11) and estimate the error. By (2.10), we have $F(t) \Xi=\Xi+t F_{1} \Xi+\mathcal{J}_{3}$, where $F_{1}$ is the operator defined by (2.15), and $\mathcal{J}_{3}=F_{2}(t) \Xi$ satisfies the estimate

$$
\left\|\mathcal{J}_{3}\right\| \leq \beta^{(2)} t^{2}\left(c_{*} t^{2}+\varepsilon^{2}\right)^{-1} \delta^{-1}\left\|X_{1}\right\|^{2} \leq \beta^{(2)} c_{*}^{-1} \delta^{-1}\left\|X_{1}\right\|^{2}=: C_{3} .
$$

Next, $F_{1} \Xi=Z \Xi+P Z^{*} P \Xi=Z \Xi$, so that (5.11) takes the form

$$
\mathcal{G}=\Xi+t Z \Xi-t^{3} \Xi N \Xi+\mathcal{J}_{1}+\mathcal{J}_{2}+\mathcal{J}_{3} .
$$


Our next goal is to estimate the discrepancy resulting from the replacement of the operator $\mathcal{G}=F(t)\left(A(t)+\varepsilon^{2} I\right)^{-1} P$ by the operator $G=F(t)\left(A(t)+\varepsilon^{2} I\right)^{-1}$. Using (2.10) once again, we get

$$
G-\mathcal{G}=F(t)\left(A(t)+\varepsilon^{2} I\right)^{-1}(F(t)-P)=t F(t)\left(A(t)+\varepsilon^{2} I\right)^{-1} F_{1}+\mathcal{J}_{3}^{\prime} .
$$

By $(2.10)$ and (5.3), the operator $\mathcal{J}_{3}^{\prime}=F(t)\left(A(t)+\varepsilon^{2} I\right)^{-1} F_{2}(t)$ admits the same estimate as $\mathcal{J}_{3}$ :

$$
\left\|\mathcal{J}_{3}^{\prime}\right\| \leq C_{3} .
$$

We transform the first term on the right in (5.16) as follows:

$$
t F(t)\left(A(t)+\varepsilon^{2} I\right)^{-1} F_{1}=t \Xi F_{1}+\mathcal{J}_{4},
$$

where, by (4.15) and (5.7), the operator $\mathcal{J}_{4}$ satisfies the estimate

$$
\left\|\mathcal{J}_{4}\right\| \leq \mathcal{C}_{0} t^{2}\left(c_{*} t^{2}+\varepsilon^{2}\right)^{-1}(2 \delta)^{-1 / 2}\left\|X_{1}\right\| \leq \mathcal{C}_{0} c_{*}^{-1}(2 \delta)^{-1 / 2}\left\|X_{1}\right\|=: C_{4} .
$$

Next, we have $\Xi F_{1}=\Xi(P Z) P+\Xi P Z^{*}=\Xi Z^{*}$ by (2.15). As a result, the operator $G=F(t)\left(A(t)+\varepsilon^{2} I\right)^{-1}$ can be written as

$$
G=\mathcal{G}+t \Xi Z^{*}+\mathcal{J}_{3}^{\prime}+\mathcal{J}_{4}
$$

Combining this with (5.15), we obtain

$$
\left(A(t)+\varepsilon^{2} I\right)^{-1} F(t)=\Xi+t\left(Z \Xi+\Xi Z^{*}\right)-t^{3} \Xi N \Xi+\mathcal{J}_{1}+\mathcal{J}_{2}+\mathcal{J}_{3}+\mathcal{J}_{3}^{\prime}+\mathcal{J}_{4} .
$$

5.3. Threshold approximation. Our main result about approximation of the resolvent $R_{-\varepsilon^{2}}(t)$ near the spectral threshold $\lambda=0$ is deduced from (5.19): it suffices to note that, by (1.2), the operator $\left(A(t)+\varepsilon^{2} I\right)^{-1} F(t)^{\perp}=: \mathcal{J}_{5}$ satisfies the estimate

$$
\left\|\mathcal{J}_{5}\right\| \leq(3 \delta)^{-1}, \quad|t| \leq t^{0}(\delta) .
$$

Thus, we arrive at the following theorem.

Theorem 5.1. For $|t| \leq t^{0}(\delta)=\delta^{1 / 2}\left\|X_{1}\right\|^{-1}$ and $\varepsilon>0$, we have

$$
\left(A(t)+\varepsilon^{2} I\right)^{-1}=\Xi+t\left(Z \Xi+\Xi Z^{*}\right)-t^{3} \Xi N \Xi+\mathcal{J} .
$$

Here $\Xi=\Xi(t, \varepsilon)=\left(t^{2} S P+\varepsilon^{2} I\right)^{-1} P$; the operators $Z$ and $N$ are defined by (1.5) and (4.13), respectively. The operator-valued function $\mathcal{J}=\mathcal{J}_{1}+\mathcal{J}_{2}+\mathcal{J}_{3}+\mathcal{J}_{3}^{\prime}+\mathcal{J}_{4}+\mathcal{J}_{5}$ is uniformly bounded:

$$
\|\mathcal{J}\| \leq C_{1}+C_{2}+2 C_{3}+C_{4}+(3 \delta)^{-1}=: C,
$$

where the constants $C_{1}, C_{2}, C_{3}$, and $C_{4}$ are defined by (5.12), (5.13), (5.14), and (5.18), respectively.

It may happen that $Z=0$, and then also $N=0$. In such cases the following theorem is useful.

Theorem 5.2. Under the assumptions of Theorem 5.1, suppose in addition that $Z=0$. Then relations (5.21) and (5.22) turn into the estimate

$$
\left\|\left(A(t)+\varepsilon^{2} I\right)^{-1}-\left(t^{2} S P+\varepsilon^{2} I\right)^{-1} P\right\| \leq C .
$$

Remark 5.3. a) For small $\varepsilon>0$, Theorem 5.1 provides a much sharper approximation for the resolvent than the estimate

$$
\left\|\left(A(t)+\varepsilon^{2} I\right)^{-1}-\Xi\right\| \leq \mathcal{C}_{0}\left(2 c_{*}^{1 / 2} \varepsilon\right)^{-1}+(3 \delta)^{-1},
$$

which follows from (5.7) and (5.20).

b) If $Z=0$, the order of the estimate (5.23) (for small $\varepsilon>0$ ) is better than that of the universal estimate (5.24). 
5.4. Comparison of the resolvents of two operator families. Let $\widetilde{A}(t)$ be another operator family of the form (1.1), and let $\widetilde{A}(t)$ be threshold equivalent to the family $A(t)$. All the objects corresponding to this family will be marked by " $\sim$ ". Note that $\widetilde{\mathfrak{N}}=\mathfrak{N}$, $\widetilde{S}=S$, and $\widetilde{\Xi}=\Xi$. We suppose that $\widetilde{A}(t)$ satisfies the condition of the form (5.2). Furthermore, suppose that for $\widetilde{A}(t)$ we have

$$
\widetilde{Z}=0 .
$$

(In homogenization problems the family $\widetilde{A}(t)$ is related to the effective differential operator. The latter is an operator with constant coefficients, so that condition (5.25) is surely satisfied.) Then, by Theorem 5.2 ,

$$
\left\|\left(\widetilde{A}(t)+\varepsilon^{2} I\right)^{-1}-\Xi\right\| \leq \widetilde{C}, \quad|t| \leq \widetilde{t}^{0}(\widetilde{\delta})=\widetilde{\delta}^{1 / 2}\left\|\widetilde{X}_{1}\right\|^{-1} .
$$

The following theorem is a consequence of (5.23) and (5.26).

Theorem 5.4. Suppose that $A(t)$ and $\widetilde{A}(t)$ are threshold equivalent and that both families satisfy the conditions of Theorem 5.2. Then

$$
\left\|\left(A(t)+\varepsilon^{2} I\right)^{-1}-\left(\widetilde{A}(t)+\varepsilon^{2} I\right)^{-1}\right\| \leq C+\widetilde{C}
$$

whenever

$$
|t| \leq t_{\min }^{0}:=\min \left\{t^{0}(\delta), \widetilde{t}^{0}(\widetilde{\delta})\right\} .
$$

For a family $A(t)$ of general type, the operator $\Xi$ in (5.21) can be replaced by $\left(\widetilde{A}(t)+\varepsilon^{2} I\right)^{-1}$, and the corresponding error term can be estimated with the help of (5.26). For this, we only need to take inequalities (1.8), (4.16), (5.3), (5.4), (5.22), and (5.27) into account. Thus, the following statement is true.

Theorem 5.5. Suppose that $A(t)$ and $\widetilde{A}(t)$ are threshold equivalent and that condition (5.25) is satisfied. Then

$$
\begin{aligned}
\left(A(t)+\varepsilon^{2} I\right)^{-1}= & \left(\widetilde{A}(t)+\varepsilon^{2} I\right)^{-1}+t\left(Z\left(\widetilde{A}(t)+\varepsilon^{2} I\right)^{-1}+\left(\widetilde{A}(t)+\varepsilon^{2} I\right)^{-1} Z^{*}\right) \\
& -t^{3}\left(\widetilde{A}(t)+\varepsilon^{2} I\right)^{-1} N\left(\widetilde{A}(t)+\varepsilon^{2} I\right)^{-1}+\mathcal{J}_{*}, \quad|t| \leq t_{\text {min }}^{0},
\end{aligned}
$$

and

$$
\left\|\mathcal{J}_{*}\right\| \leq C+\widetilde{C}\left(1+2^{-1 / 2}+2^{-1 / 2}\left(c_{*}^{-1}+\widetilde{c}_{*}^{-1}\right)\left\|X_{1}\right\|^{2}\right)=: C_{*} .
$$

\section{§6. Approximation of the generalized Resolvent}

6.1. Preliminaries. We borrow some preliminary information from $\mathrm{BSu}$, Subsections 1.5 and 5.3]. Along with $\mathfrak{H}$, we consider another Hilbert space $\widehat{\mathfrak{H}}$ and a family $\widehat{X}(t)=$ $\widehat{X}_{0}+t \widehat{X}_{1}: \widehat{\mathfrak{H}} \rightarrow \mathfrak{H}_{*}$ of the same type as $X(t)$; the space $\mathfrak{H}_{*}$ remains the same. Let $M: \mathfrak{H} \rightarrow \widehat{\mathfrak{H}}$ be an isomorphism such that

$$
M \operatorname{Dom} X_{0}=\operatorname{Dom} \widehat{X}_{0}, \quad X(t)=\widehat{X}(t) M ;
$$

then $X_{0}=\widehat{X}_{0} M$ and $X_{1}=\widehat{X}_{1} M$. We introduce the selfadjoint family

$$
\widehat{A}(t)=\widehat{X}(t)^{*} \widehat{X}(t)
$$

in $\widehat{\mathfrak{H}}$. Then, obviously,

$$
A(t)=M^{*} \widehat{A}(t) M \text {. }
$$


In what follows, all the objects corresponding to the family $\widehat{A}(t)$ are marked by "^^" Observe that

$$
\begin{gathered}
\widehat{\mathfrak{N}}=M \mathfrak{N}, \quad \widehat{n}=n, \\
\widehat{\mathfrak{N}}_{*}=\mathfrak{N}_{*}, \quad \widehat{n}_{*}=n_{*}, \quad \widehat{P}_{*}=P_{*}, \\
R=\left.\widehat{R} M\right|_{\mathfrak{N}}, \quad \operatorname{rank} R=\operatorname{rank} \widehat{R}, \\
S=\left.P M^{*} \widehat{S} M\right|_{\mathfrak{N}}, \quad \widehat{S}=\left.\widehat{P}\left(M^{*}\right)^{-1} S M^{-1}\right|_{\widehat{\mathfrak{N}}}
\end{gathered}
$$

(see [BSu, Subsection 1.5]). We introduce the following notation:

$$
Q:=\left(M M^{*}\right)^{-1}=\left(M^{*}\right)^{-1} M^{-1}: \widehat{\mathfrak{H}} \rightarrow \widehat{\mathfrak{H}} \text {. }
$$

The operator $Q$ is positive and continuous together with $Q^{-1}$. If an operator $Q$ with such properties is given initially (along with $\widehat{A}(t)$ ), then $Q$ admits a (nonunique) representation of the form (6.7). Then we can reconstruct the families $X(t)$ and $A(t)$ by formulas (6.1) and (6.3).

Inequality (5.2) implies that for $\widehat{A}(t)$ we have

$$
\widehat{A}(t) \geq \widehat{c}_{*} t^{2} I, \quad \widehat{c}_{*}=c_{*}\|M\|^{-2}, \quad|t| \leq t^{0} .
$$

By definition, the generalized resolvent (or $Q$-resolvent) for the family $\widehat{A}(t)$ is the following selfadjoint operator in $\widehat{\mathfrak{H}}$ :

$$
\left(\widehat{A}(t)+\varepsilon^{2} Q\right)^{-1}, \quad \varepsilon>0 .
$$

From (6.3) and (6.7) it follows that

$$
\left(\widehat{A}(t)+\varepsilon^{2} Q\right)^{-1}=M\left(A(t)+\varepsilon^{2} I\right)^{-1} M^{*}
$$

(see $[\mathrm{BSu},(5.13)])$. Next, let $Q_{\widehat{\mathfrak{N}}}$ be the block of the operator $Q$ in the subspace $\widehat{\mathfrak{N}}$ :

$$
Q_{\widehat{\mathfrak{N}}}=\left.\widehat{P} Q\right|_{\widehat{\mathfrak{N}}}: \widehat{\mathfrak{N}} \rightarrow \widehat{\mathfrak{N}} .
$$

By (5.2), the germ $S$ is nondegenerate, whence, by (6.6), the germ $\widehat{S}$ is also nondegenerate. Then the operator

$$
\left(t^{2} \widehat{S}+\varepsilon^{2} Q_{\widehat{\mathfrak{N}}}\right)^{-1}: \widehat{\mathfrak{N}} \rightarrow \widehat{\mathfrak{N}}
$$

makes sense, and (see $[\mathrm{BSu},(5.18)])$ we have

$$
M \Xi M^{*}=M\left(t^{2} S P+\varepsilon^{2} I\right)^{-1} P M^{*}=\left(t^{2} \widehat{S}+\varepsilon^{2} Q_{\widehat{\mathfrak{N}}}\right)^{-1} \widehat{P}=: \widehat{\Xi}_{Q} .
$$

6.2. Approximation for the $Q$-resolvent of $\widehat{A}(t)$. Relations (5.21) and (6.10) imply the following representation for the operator $(6.9)$ :

$$
\begin{aligned}
\left(\widehat{A}(t)+\varepsilon^{2} Q\right)^{-1}= & M \Xi M^{*}+t M\left(Z \Xi+\Xi Z^{*}\right) M^{*} \\
& -t^{3} M \Xi N \Xi M^{*}+M \mathcal{J} M^{*}, \quad|t| \leq t^{0} .
\end{aligned}
$$

Using (6.12), we can rewrite (6.13) as

$$
\begin{aligned}
\left(\widehat{A}(t)+\varepsilon^{2} Q\right)^{-1}= & \widehat{\Xi}_{Q}+t\left(M Z M^{-1} \widehat{P} \widehat{\Xi}_{Q}+\widehat{\Xi}_{Q} \widehat{P}\left(M^{*}\right)^{-1} Z^{*} M^{*}\right) \\
& -t^{3} \widehat{\Xi}_{Q} \widehat{P}\left(M^{*}\right)^{-1} N M^{-1} \widehat{P} \widehat{\Xi}_{Q}+M \mathcal{J} M^{*} .
\end{aligned}
$$

Lemma 6.1. Let $\widehat{Z}_{Q}$ be the operator in $\widehat{\mathfrak{H}}$ that takes $\widehat{u} \in \widehat{\mathfrak{H}}$ to a unique solution $\widehat{\psi}_{Q} \in \widehat{\mathfrak{H}}$ of the equation

$$
\widehat{X}_{0}^{*}\left(\widehat{X}_{0} \widehat{\psi}_{Q}+\widehat{X}_{1} \widehat{\omega}\right)=0, \quad Q \widehat{\psi}_{Q} \perp \widehat{\mathfrak{N}}
$$

where $\widehat{\omega}=\widehat{P} \widehat{u} \in \widehat{\mathfrak{N}}$. Then

$$
M Z M^{-1} \widehat{P}=\widehat{Z}_{Q} .
$$


Proof. The proof reduces to an elementary recalculation in equation (1.3), which defines the operator $Z$ under the condition (1.4) (see (1.5)). We only need to take (6.1), (6.4), and (6.7) into account.

Observe that (6.16) and (1.8) imply the estimate

$$
\left\|\widehat{Z}_{Q}\right\| \leq(8 \delta)^{-1 / 2}\left\|X_{1}\right\|\|M\|\left\|M^{-1}\right\| .
$$

In order to transform the representation (6.14), we also need the following lemma.

Lemma 6.2. The following identity is true:

$$
\widehat{P}\left(M^{*}\right)^{-1} N M^{-1} \widehat{P}=\widehat{Z}_{Q}^{*} \widehat{X}_{1}^{*} \widehat{R} \widehat{P}+(\widehat{R} \widehat{P})^{*} \widehat{X}_{1} \widehat{Z}_{Q}=: \widehat{N}_{Q} .
$$

Proof. By (4.13), (6.1), and (6.16), we have

$$
\begin{aligned}
\widehat{P}\left(M^{*}\right)^{-1} N M^{-1} \widehat{P} & =\widehat{Z}_{Q}^{*}\left(M^{*}\right)^{-1} X_{1}^{*}(R P) M^{-1} \widehat{P}+\widehat{P}\left(M^{*}\right)^{-1}(R P)^{*} X_{1} M^{-1} \widehat{Z}_{Q} \\
& =\widehat{Z}_{Q}^{*} \widehat{X}_{1}^{*}(R P) M^{-1} \widehat{P}+\widehat{P}\left(M^{*}\right)^{-1}(R P)^{*} \widehat{X}_{1} \widehat{Z}_{Q} .
\end{aligned}
$$

Next, by (6.5), $R P=\widehat{R} M P$, and by (6.4), $R P M^{-1} \widehat{P}=\widehat{R} M M^{-1} \widehat{P}=\widehat{R} \widehat{P}$. Substituting this in (6.19), we arrive at (6.18).

For the operator $\widehat{N}_{Q}$ inequality (6.17) implies the estimate

$$
\left\|\widehat{N}_{Q}\right\| \leq(2 \delta)^{-1 / 2}\left\|X_{1}\right\|\|M\|\left\|M^{-1}\right\|\left\|\widehat{X}_{1}\right\|^{2} .
$$

Substituting (6.16) and (6.18) in (6.14), we obtain the following statement.

Theorem 6.3. For the operator (6.9) we have

$$
\left(\widehat{A}(t)+\varepsilon^{2} Q\right)^{-1}=\widehat{\Xi}_{Q}+t\left(\widehat{Z}_{Q} \widehat{\Xi}_{Q}+\widehat{\Xi}_{Q} \widehat{Z}_{Q}^{*}\right)-t^{3} \widehat{\Xi}_{Q} \widehat{N}_{Q} \widehat{\Xi}_{Q}+\mathcal{J}_{M} .
$$

Here the operator $\widehat{\Xi}_{Q}$ is defined by (6.11) and (6.12), $\widehat{Z}_{Q}$ is the operator introduced in Lemma 6.1 (see equation (6.15)), and the operator $\widehat{N}_{Q}$ is given by the right-hand side of (6.18). The operator $\mathcal{J}_{M}:=M \mathcal{J} M^{*}$ satisfies the estimate

$$
\left\|\mathcal{J}_{M}\right\| \leq C\|M\|^{2}, \quad|t| \leq t^{0}
$$

where the constant $C$ is defined by (5.22).

Remark 6.4. The representation (6.21) is written in terms of the family $\widehat{A}(t)$ defined by (6.2). Only the constants $C$ and $t^{0}$ in (6.22) are defined in terms of the family $A(t)$. They can also be recalculated in terms of $\widehat{A}$, though, in general, with some loss. For instance, a possible value of $\widehat{c}_{*}$ is given in (6.8), and we can take $\widehat{t}^{0}=t^{0}\|M\|^{-1}\left\|M^{-1}\right\|^{-1}$. We shall not discuss this in detail here.

Theorem 6.5. Under the conditions of Theorem 6.3, suppose that

$$
\widehat{Z}_{Q}=0
$$

(which is equivalent to the condition $Z=0$ ). Then relations (6.21) and (6.22) imply the estimate

$$
\left\|\left(\widehat{A}(t)+\varepsilon^{2} Q\right)^{-1}-\left(t^{2} \widehat{S}+\varepsilon^{2} Q_{\widehat{\mathfrak{N}}}\right)^{-1} \widehat{P}\right\| \leq C\|M\|^{2}, \quad|t| \leq t^{0} .
$$


6.3. Another approximation for the $Q$-resolvent (6.9). Now, let $\widehat{A}_{+}(t)$ be another family of the form (6.2), acting in $\widehat{\mathfrak{H}}$, and let $Q_{+}$be a bounded positive definite operator in $\widehat{\mathfrak{H}}$. We put $M_{+}=\left(Q_{+}\right)^{-1 / 2}$ and introduce the operator family

$$
A_{+}(t)=M_{+} \widehat{A}_{+}(t) M_{+} .
$$

Suppose that $A_{+}(t)$ satisfies the analog of condition (5.2) with constant $c_{*}^{(+)}$for $|t| \leq t_{+}^{0}$. In accordance with (6.8), we put

$$
\widehat{c}_{*}^{(+)}=c_{*}^{(+)}\left\|M_{+}\right\|^{-2} .
$$

Moreover, we assume that the following condition is satisfied.

Condition 6.6. 1) The families $\widehat{A}(t)$ and $\widehat{A}_{+}(t)$ are threshold equivalent. 2) The blocks of the operators $Q$ and $Q_{+}$in $\widehat{\mathfrak{N}}=\widehat{\mathfrak{N}}_{+}$coincide. 3) The operator $Z_{+}$corresponding to the family $A_{+}(t)$ is equal to zero: $Z_{+}=0$ (and then also $\widehat{Z}_{+, Q_{+}}=0$ ).

We mention the following simple estimates:

$$
\begin{aligned}
\left\|\left(\widehat{A}_{+}(t)+\varepsilon^{2} Q_{+}\right)^{-1}\right\| & \leq\left(\widehat{c}_{*}^{(+)}\right)^{-1} t^{-2}, \quad|t| \leq t_{+}^{0}, \\
\left\|\left(t^{2} \widehat{S}+\varepsilon^{2} Q_{\widehat{\mathfrak{N}}}\right)^{-1} \widehat{P}\right\| & \leq\left(\widehat{c}_{*}^{(+)}\right)^{-1} t^{-2} .
\end{aligned}
$$

Theorem 6.5 applies to the family $\widehat{A}_{+}(t)$. Thus, using the notation (6.12), we have

$$
\begin{aligned}
\left(\widehat{A}_{+}(t)+\varepsilon^{2} Q_{+}\right)^{-1} & =\widehat{\Xi}_{Q}+\mathcal{J}_{M_{+}}, \\
\left\|\mathcal{J}_{M_{+}}\right\| \leq C_{+}\left\|M_{+}\right\|^{2} & =C_{+}\left\|Q_{+}^{-1}\right\|, \quad|t| \leq t_{+}^{0} .
\end{aligned}
$$

Here $C_{+}$is the constant of the form (5.22) corresponding to the family $A_{+}(t)$. Relation (6.25) allows us to eliminate $\widehat{\Xi}_{Q}$ in (6.21). As a result, we obtain

$$
\begin{aligned}
\left(\widehat{A}(t)+\varepsilon^{2} Q\right)^{-1}= & \left(\widehat{A}_{+}(t)+\varepsilon^{2} Q_{+}\right)^{-1} \\
& +t \widehat{Z}_{Q}\left(\widehat{A}_{+}(t)+\varepsilon^{2} Q_{+}\right)^{-1}+t\left(\widehat{A}_{+}(t)+\varepsilon^{2} Q_{+}\right)^{-1} \widehat{Z}_{Q}^{*} \\
& -t^{3}\left(\widehat{A}_{+}(t)+\varepsilon^{2} Q_{+}\right)^{-1} \widehat{N}_{Q}\left(\widehat{A}_{+}(t)+\varepsilon^{2} Q_{+}\right)^{-1}+\check{\mathcal{J}} .
\end{aligned}
$$

Here

$$
\begin{gathered}
\check{\mathcal{J}}=\mathcal{J}_{M}-\mathcal{J}_{M_{+}}-t\left(\widehat{Z}_{Q} \mathcal{J}_{M_{+}}+\mathcal{J}_{M_{+}} \widehat{Z}_{Q}^{*}\right) \\
+t^{3}\left(\widehat{A}_{+}(t)+\varepsilon^{2} Q_{+}\right)^{-1} \widehat{N}_{Q} \mathcal{J}_{M_{+}}+t^{3} \mathcal{J}_{M_{+}} \widehat{N}_{Q}\left(t^{2} \widehat{S}+\varepsilon^{2} Q_{\widehat{\mathfrak{N}}}\right)^{-1} \widehat{P} \\
|t| \leq t_{\min }^{0}:=\min \left\{t^{0}, t_{+}^{0}\right\}
\end{gathered}
$$

The operator $\check{\mathcal{J}}$ is estimated with the help of (6.17), (6.20), (6.23), (6.24), and (6.29). We should also recall (6.22) and (6.26). This leads to the estimate

$$
\begin{aligned}
\|\check{\mathcal{J}}\| \leq & C\|M\|^{2} \\
& +C_{+}\left\|Q_{+}^{-1}\right\|\left(1+2^{-1 / 2}\|M\|\left\|M^{-1}\right\|\left(1+2\left(\widehat{c}_{*}^{(+)}\right)^{-1}\left\|\widehat{X}_{1}\right\|^{2}\right)\right), \quad|t| \leq t_{\min }^{0}
\end{aligned}
$$

As a result, we arrive at the following statement.

Theorem 6.7. Let $A(t), \widehat{A}(t)$, and $\widehat{A}_{+}(t)$ be the families defined above. Suppose that (6.3) and Condition 6.6 are satisfied. Then the representation (6.27) and estimate (6.30) are valid. 
6.4. Representation of the resolvent $\left(A(t)+\varepsilon^{2} I\right)^{-1}$. A representation for the resolvent $\left(A(t)+\varepsilon^{2} I\right)^{-1}$ follows immediately from Theorem 6.7 and identity (6.10).

Theorem 6.8. Under the conditions of Theorem 6.7, we have

$$
\begin{aligned}
\left(A(t)+\varepsilon^{2} I\right)^{-1}=M^{-1}\left(\widehat{R}_{+}(t, \varepsilon)+\right. & t\left(\widehat{Z}_{Q} \widehat{R}_{+}(t, \varepsilon)+\widehat{R}_{+}(t, \varepsilon) \widehat{Z}_{Q}^{*}\right) \\
& \left.-t^{3} \widehat{R}_{+}(t, \varepsilon) \widehat{N}_{Q} \widehat{R}_{+}(t, \varepsilon)+\check{\mathcal{J}}\right)\left(M^{*}\right)^{-1},
\end{aligned}
$$

where

$$
\widehat{R}_{+}(t, \varepsilon)=\left(\widehat{A}_{+}(t)+\varepsilon^{2} Q_{+}\right)^{-1},
$$

and the operator-valued function (6.28) is estimated in (6.30).

6.5. Approximation of the generalized resolvent of the family $A(t)$. In conclusion, we carry Theorem 6.8 over to the case of the generalized resolvent

$$
\left(A(t)+\varepsilon^{2} \mathfrak{Q}\right)^{-1},
$$

where $\mathfrak{Q}: \mathfrak{H} \rightarrow \mathfrak{H}$ is a continuous positive definite operator. We may assume that the operator $\mathfrak{Q}$ is written in the form

$$
\mathfrak{Q}=\left(\mathfrak{M M}^{*}\right)^{-1},
$$

where $\mathfrak{M}: \mathfrak{H} \rightarrow \mathfrak{H}$ is an operator bounded together with its inverse (for instance, we can put $\left.\mathfrak{M}=\mathfrak{Q}^{-1 / 2}\right)$. Suppose that the family $\widehat{A}(t)$ and the operator $M$ are as in Subsection 6.1 ; in particular, we assume (6.3). Then, by (6.33),

$$
\left(A(t)+\varepsilon^{2} \mathfrak{Q}\right)^{-1}=M^{-1}\left(\widehat{A}(t)+\varepsilon^{2} Q^{\nabla}\right)^{-1}\left(M^{*}\right)^{-1},
$$

where

$$
Q^{\nabla}=\left(M^{\nabla}\left(M^{\nabla}\right)^{*}\right)^{-1}, \quad M^{\nabla}:=M \mathfrak{M} .
$$

Now, suppose that the family $\widehat{A}_{+}(t)$ and the operator $Q_{+}^{\nabla}$ satisfy the conditions of Subsection 6.3 (and, in particular, Condition 6.6) with $Q$ replaced by $Q^{\nabla}$ and $Q_{+}$replaced by $Q_{+}^{\nabla}$. Then (6.27) is true with $Q$ replaced by $Q^{\nabla}$ and $Q_{+}$replaced by $Q_{+}^{\nabla}$. However, the remainder term will change, and we change its notation. Moreover, we denote $A^{\nabla}(t):=$ $\left(M^{\nabla}\right)^{*} \widehat{A}(t) M^{\nabla}$ and $A_{+}^{\nabla}(t):=M_{+}^{\nabla} \widehat{A}_{+}(t) M_{+}^{\nabla}$, where $M_{+}^{\nabla}=\left(Q_{+}^{\nabla}\right)^{-1}$. Let $t^{\nabla}$ play the role of $t^{0}$ for $A^{\nabla}(t)$, and let $t_{+}^{\nabla}$ play the role of $t^{0}$ for $A_{+}^{\nabla}(t)$. As a result, we arrive at the following statement.

Theorem 6.9. Under the above assumptions, the $\mathfrak{Q}$-resolvent (6.32) admits the representation

$$
\left(A(t)+\varepsilon^{2} \mathfrak{Q}\right)^{-1}=M^{-1}\left(\widehat{R}_{+}^{\nabla}(t, \varepsilon)+t\left(\widehat{Z}_{Q^{\nabla}} \widehat{R}_{+}^{\nabla}(t, \varepsilon)+\widehat{R}_{+}^{\nabla}(t, \varepsilon) \widehat{Z}_{Q^{\nabla}}^{*}\right)\right.
$$

$$
\left.-t^{3} \widehat{R}_{+}^{\nabla}(t, \varepsilon) \widehat{N}_{Q \nabla} \widehat{R}_{+}^{\nabla}(t, \varepsilon)\right)\left(M^{*}\right)^{-1}+\mathcal{J}^{\nabla}
$$

where $\widehat{R}_{+}^{\nabla}(t, \varepsilon):=\left(\widehat{A}_{+}(t)+\varepsilon^{2} Q_{+}^{\nabla}\right)^{-1}$, and the norm of the operator-valued function $\mathcal{J}^{\nabla}=$ $\mathcal{J}^{\nabla}(t, \varepsilon)$ is uniformly bounded for $|t| \leq \min \left\{t^{\nabla}, t_{+}^{\nabla}\right\}, \varepsilon>0$.

An explicit estimate for $\left\|\mathcal{J}^{\nabla}\right\|$ can be obtained in the same way as for (6.30), but we shall not dwell on this.

If $\mathfrak{Q}=I$, then (6.34) coincides with (6.31). 


\section{REFERENCES}

[BSu] M. Sh. Birman and T. A. Suslina, Second order periodic differential operators. Threshold properties and homogenization, Algebra i Analiz 15 (2003), no. 5, 1-108; English transl., St. Petersburg Math. J. 15 (2004), no. 5, 639-714. MR2068790(2005k:47097)

[K] T. Kato, Perturbation theory for linear operators, Springer-Verlag, Berlin, 1995. MR.1335452 (96a:47025)

Department of Physics, St. Petersburg State University, Ulyanovskaya 1, Petrodvorets, St. Petersburg 198504, Russia

E-mail address: mbirman@list.ru

Department of Physics, St. Petersburg State University, Ulyanovskaya 1, Petrodvorets, St. Petersburg 198504, Russia

E-mail address: suslina@list.ru

Received 11/APR/2005

Translated by T. A. SUSLINA 